This is an author produced version of a paper published in Biochimica et Biophysica Acta. This paper has been peer-reviewed but does not include the final publisher proof-corrections or journal pagination.

Citation for the published paper:

Löfgren, Lars.

"Down-regulation of apolipoprotein $\mathrm{M}$ expression is mediated by Phosphatidylinositol 3-Kinase in HepG2 Cells"

Biochimica et Biophysica Acta, 2006, Vol: 1761, Issue: 2, pp. 256-60. http://dx.doi.org/10.1016/j.bbalip.2006.02.002

Access to the published version may require journal subscription. Published with permission from: Elsevier 


\title{
Down-regulation of apolipoprotein $M$ expression is mediated by Phosphatidylinositol 3-Kinase in HepG2 Cells
}

\author{
Ning $\mathrm{Xu}^{1 *}$, Bo Ahrén ${ }^{2}$, Jingting Jiang ${ }^{1,3}$ and Peter Nilsson-Ehle ${ }^{1}$ \\ ${ }^{1}$ Department of Laboratory Medicine and ${ }^{2}$ Department of Medicine, Lunds University, S- \\ 22185 Lund, Sweden; and ${ }^{3}$ Department of Molecular Laboratory, the third Affiliated \\ Hospital of Suzhou University, Changzhou 213003, China
}

Running title: Insulin inhibits apoM in HepG2 cells

*Corresponding person:

Ning Xu, MD, PhD

Section of Clinical Chemistry \& Pharmacology

Department of Laboratory Medicine

Lunds University

S-221 85 Lund, Sweden

Tel. +46-46173462

Fax. +46-46130064

Email.ning.xu@med.lu.se 


\begin{abstract}
Apolipoprotein M (apoM) is a novel apolipoprotein present mostly in high-density lipoprotein (HDL) in human plasma. In the present study, we demonstrate that insulin, insulin-like growth factor I (IGF-I), and IGF-I potential peptide (IGF-IPP) significantly inhibits apoM expression, in a dose- and a time-dependent manner, in the human hepatoma cell line, HepG2 cells. Insulin-induced down-regulation of apoM was blocked by AG1024 (a specific insulin receptor inhibitor) and LY294002 (a phsophatidylinositol 3-kinase (PI3K) inhibitor), which indicates that it is mediated via the activation of PI3K pathway. In contrast, PD98059 (a MAP kinase inhibitor) did not influence insulininduced down-regulation of apoM expression, and activation of neither PPAR- $\alpha$ agonist (GW7647) nor PPAR- $\gamma$ agonist (GW1929) influences apoM expression in HepG2 cells, which indicates that regulation of apoM expression is not related to the activation of PPAR- $\alpha$ and PPAR- $\gamma$ in hepatic cells, whereas, both PPAR- $\alpha$ and PPAR- $\gamma$ agonists could inhibit apoB expression. Moreover, in the present study we demonstrated that PPAR $\beta / \delta$ agonist (GW501516) could inhibit both apoM and apoB expression in the HepG2 cells. In conclusion, this study shows that apoM expression is regulated by PI3-kinase in HepG2-cells.
\end{abstract}

Key words: Apolipoprotein M; Insulin; phsophatidylinositol 3-kinase and HepG2 cell line; PPARs. 


\section{Introduction}

Apolipoprotein M (apoM) is a recently discovered human apolipoprotein that is associated mainly with high-density lipoprotein (HDL) in human plasma. It is also present in triglyceride-rich lipoproteins (TGRLP) and low-density lipoprotein (LDL) [1, 2]. ApoM is mainly expressed in hepatocytes and in the tubular epithelial cells in kidney, although small amounts are detected also in fetal liver and kidney [3, 4]. It was recently demonstrated that apoM is important for pre $\beta$-HDL formation and may have protective effect on atherosclerosis [5]. Hence, mice with apoM deficiency showed impaired pre $\beta$ HDL formation and further overexpression of apoM in LDL-receptor knock-out mice protected against atherosclerosis when fed a high cholesterol diet [5]. In our previous studies we have demonstrated that the proportion of apoM in TGRLP increases in the postprandial phase [1]. Moreover, we have demonstrated that leptin signaling is essential for the apoM synthesis in vivo, because in the leptin deficient (ob/ob) mouse and leptin receptor deficient $(d b / d b)$ mouse apoM mRNA levels in liver and kidney and plasma apoM concentrations were greatly reduced when compared to the normal mouse [6]. We have also demonstrated that plasma apoM concentration is positively correlated to leptin level in obese subjects [7]. In addition, apoM, like apoB, could be regulated by several cytokines in a hepatic cell line, HepG2 cells [8-11]. However, the pathophysiological importance of apoM in human is still unknown

To further understand the mechanism of apoM expression, we have investigated the effects of insulin, insulin like growth factor I (IGF-I), and IGF-I potential peptide (IGF- 
IPP) on the regulation of apoM expression in HepG2 cells. Furthermore we examined possible pathways of inhibitory effects of insulin on apoM expression in the cells. 


\section{Material and methods}

\section{Materials}

Insulin was purchased from Nova Nordisk (Bagsvaerd, Denmark). IGF-IPP was

purchased from Immunological and Biochemical Testsystems (Reutlingen, Germany). IGF-I, PPAR- $\alpha$ agonist (GW7647), PPAR- $\gamma$ agonist (GW1929) and LY294002 were purchased from Sigma-Aldrich (St. Louis, MO, USA). PPAR- $\beta / \delta$ agonist (GW501516) was purchased from Alexis Biochemicals, USA. AG1024 and PD98058 were obtained from EMD Biosciences, Germany. The established hepatoblastoma cell line, HepG2, was from American Type Culture Collection (Manassas, VA, USA). Six-well cell culture clusters and $25-\mathrm{cm}^{2}$ vented cell culture flasks were purchased from Nunc. Cell culture media were from the Invitrogen. Real-time RT-PCR reagents and control probe GAPDH were purchased from the Applied Biosystems Inc., USA.

\section{Cell cultures}

HepG2 cells were cultured in 25-cm² vented flasks containing RPMI 1640 with $10 \%$ fetal calf serum (FCS) in the presence of benzylpenicillin (100 U/ml) and streptomycin (100 $\mu \mathrm{g} / \mathrm{ml})$ under standard culture conditions $\left(5 \% \mathrm{CO}_{2}, 37^{\circ} \mathrm{C}\right)$. Cells were seeded in six-well cell culture clusters, and were grown to 50-70\% confluence. Prior to experiment, cells were washed twice with phosphate buffered saline (PBS), and once with serum-free RPMI 1640 without antibiotic. Experimental medium contained RPMI 1640 with 0.5\% human serum albumin (HSA) and one or more additives, i.e., insulin, IGF-I, IGF-IPP, PPAR- $\alpha$, PPAR- $\gamma$, et al, at different concentrations added as described in the legend to figures.

Isolation of total RNA from HepG2 cells and real-time RT-PCR analyses 
Total RNA of HepG2 cells was isolated by the guanidinium thiocyanate method [12]. Primer Express software (Applied Biosystems) was used to design the human apoB and apoM primers and probes used in the TaqMan assay. The primers and probes are the same as described previously [10]. A commercial GAPDH primer/probe set (Applied Biosystems) was used in separate tubes as control for the input cDNA. Relative standard curves for apoM and GAPDH were performed to compensate the efficiency of PCR. A serial dilution of human apoM cDNA was used to generate a standard curve by plotting the cycle threshold versus the log of input cDNA. The apoM and GAPDH standards were linear input cDNA [10]. Quantification of apoB mRNA levels or apoM mRNA levels is relative to GAPDH mRNA levels, and was performed on an ABI PRISM 7700 Sequence Detector (Applied Biosystems), as described previously [10]. In brief, real-time RT-PCR was performed in two steps. It was carried out in a 25 - $\mu$ l reaction mixture containing $1 \mu \mathrm{l}$ TaqMan Universal PCR Master Mix, 22.5 pmol of both forward and reverse primers, 5 pmol probes and 50 ng of the total RNA templates. Thermal cycling conditions included the following steps: $25^{\circ} \mathrm{C} 10 \mathrm{~min}, 48{ }^{\circ} \mathrm{C} 30 \mathrm{~min}$ and $95{ }^{\circ} \mathrm{C} 5 \mathrm{~min}$ to do reverse transcription, and then the reaction mixture was preheated for 2 min at $50{ }^{\circ} \mathrm{C}$ and for 10 min at $95{ }^{\circ} \mathrm{C}$ to activate Taq polymerase. After that, a 40-cycle two-step PCR was performed consisting of $15 \mathrm{~s}$ at $95{ }^{\circ} \mathrm{C}$ and $1 \mathrm{~min}$ at $60{ }^{\circ} \mathrm{C}$. Samples were amplified simultaneously in triplicates in one-assay run. Real-time RT-PCR of GAPDH was performed according to the manufacturer's instruction. The threshold cycle (CT) is defined as the fractional cycle number at which the reporter fluorescence reaches a certain level. 


\section{Statistics}

Results are expressed as means \pm SD. Comparisons among groups were statistically analyzed by the one-way ANOVA and followed by the unpaired Student's $t$-test. A $p$ value less then 0.05 is considered as significant. 


\section{Results}

Effects of insulin, IGF-I or IGF-IPP on apoM expression

As shown in figures 1-3, apoM mRNA levels were significantly suppressed by administration of insulin, IGF-I or IGF-IPP in a dose-dependent manner. At $0.1 \mu \mathrm{g} / \mathrm{ml}$ insulin, apoM mRNA levels were decreased by $42 \%$ compared to controls, and at $10 \mu \mathrm{g}$ /ml insulin, apoM mRNA levels decreased by more than 60\% (Fig. 1). The IGF-I and IGF-IPP induced inhibition of apoM expression were a litter weaker than that induced by the insulin (Figs 2 and 3). Insulin induced down-regulation of apoM expression was also time-dependent and reached a maximum after 24 hours of incubation (Fig. 4). In the present study we also examined effects of insulin, IGF-I and IFG-IPP on apoB expression in HepG2 cells. Both insulin, IGF-I and IGF-IPP could significantly inhibit apoB expression in the HepG2 cells too (data not shown), which agrees to the reports in literatures [13-16].

Insulin induced inhibition of apoM expression is mediated via PI3K pathway AG1024 (an insulin receptor inhibitor) would block insulin-induced inhibition of apoM expression in HepG2 cells (Fig. 5). It is also demonstrated that LY294002 (a PI3K inhibitor) could block the insulin-induced down-regulation of apoM expression in a dose dependent manner (Fig. 6). However, down-regulation of apoM expression by insulin could not be blocked by addition of PD98058 $(50 \mu \mathrm{M})$ that is a MAP kinase inhibitor (data not shown). 
Effects of PPAR- $\alpha$, PPAR- $\gamma$ and PPAR- $\beta / \delta$ agonists on apoM and apoB expression in HepG2 cells

There were no effects of activation of PPAR- $\alpha$ (GW7647) or PPAR- $\gamma$ (GW1929) on the regulation of apoM expression in HepG2 cell cultures (Fig. 7), whereas apoB mRNA levels were significantly decreased by the administration of GW7647 and GW1929. As shown in the Fig. 8, PPAR- $\beta / \delta$ agonist (GW501516) cold inhibit both apoM and apoB expressions in HepG2 cell cultures. 


\section{Discussions}

In the present study we demonstrate that apoM expression is regulated by insulin and IGF-I in HepG2 cells in a dose- and time-dependent manner and that inhibition of apoM expression by insulin is mediated by the PI3K pathway. In contrast, MAP kinase pathway did not affect ApoM expression. Furthermore we demonstrate that activation of PPAR- $\alpha$ or PPAR- $\gamma$ does not influence apoM expression, whereas activation of PPAR- $\alpha$ and PPAR- $\gamma$ inhibits apoB expression in the HepG2 cells. However, PPAR- $\beta / \delta$ against, GW501516, could inhibit both apoM and apoB expression in Hepg2 cell cultures.

ApoM is a 26-kDa protein that is mainly associated with HDL in human plasma, with a small proportion present in TGRLP and LDL [1]. It has been demonstrated that apoM can be regulated by several biological factors, such as TGF- $\beta$, PAF and leptin $[6,9,10]$. Nevertheless, the exact mechanisms of apoM expression regulated by these factors are still unknown. It has previously been reported that insulin or IGF-I is a down-regulator of apoB synthesis $[13,14,16,17]$, which is partly via the activation of PI3K pathway [18]. It has been recently reported that PPAR- $\beta / \delta$ agonist could increase the phosphorylation of Akt [19]. In the present study we used PPAR- $\beta / \delta$ agonist (GW501516) to stimulate HepG2 cells. It demonstrated that GW501516 could inhibit apoM expression, which indicate that Akt may be also involved in the insulin-induced inhibition of apoM expression in HepG2 cells. IGF-I administration reduces insulin secretion, total triglycerides and VLDL triglycerides, LDL cholesterol and lipoprotein (a) levels in healthy and diabetic subjects [20-23]. Previous studies showed that apoB mRNA levels could be slightly decreased (16\% of controls) by administration of insulin [24], whereas 
insulin dramatically decreased apoB secretion in hepatic cells in vitro [13-16], which is mainly related to the post-translation level $[25,26]$. Our results indicate that insulin, IGFI and IGF-IPP down-regulate apoM expression in a dose- and time-dependent manner in hepatic cells. In our previously study we reported that apoM expression was significantly inhibited in the insulin resistance / hyperinsulinemia animal models (ob/ob mouse and $d b / d b$ mouse) [6]. The plasma insulin levels were 38 folds and 140 folds higher in $o b / o b$ mouse and $d b / d b$ mouse respectively, compared to the normal mouse. After short-term leptin treatment of $o b / o b$ mouse, hepatic apoM expression and plasma apoM levels were increased. At meanwhile, plasma insulin levels were significantly decreased. It is indicated that insulin may partly function as the regulator of hepatic apoM expression in vivo too.

It has been well known that PPARs regulate lipoprotein and apolipoproteins in vivo and in vitro. Fibrates are widely used drugs those lower triglycerides and increase HDL concentrations in serum. Fibrates could repress apoCIII gene expression, an effect that explains partially the triglyceride-lowering activity of these drugs [27], which is most likely via the activation of PPAR- $\alpha$. Fibrates could also increase hepatic apoA-I and apoA-II expressions [28], and stimulate cellular fatty acid uptake, conversion to acylCoA derivatives, and catabolism by the beta-oxidation pathways, which combined with a reduction in fatty acid and triglyceride synthesis, results in a decrease in VLDL production [29]. Exposure of HepG2 and Hep3B cells to gemfibrozil that is a PPAR- $\alpha$ activator results in a 2-fold induction of apoAI mRNA and a one-third reduction in apoB mRNA, but had no significant effect on apoE mRNA levels [30]. Ciprofibrate treatment 
decreases hepatic apoB mRNA editing and alters the pattern of hepatic lipoprotein secretion [31]. However, Linden et al., reported that PPAR- $\alpha$ agonist, WY 14643, increased the secretion of apoB-100, but not that of apoB-48, and decreased triglyceride biosynthesis and secretion from primary rat hepatocytes [32]. Recently, Anderson et al. reported in a micro array study demonstrated that PPAR- $\alpha$ agonist could significantly down-regulate hepatic apoM expression, but not apoB expression, in vivo [33]. In the present study we demonstrate both PPAR- $\alpha$ and PPAR- $\gamma$ agonists down-regulated apoB expression, but not apoM expression, in the HepG2 cells, which suggests that there is a different regulations of apoB and apoM expression in the hepatic cells. Moreover, PPAR$\beta / \delta$ against, GW501516, could inhibit both apoM and apoB expression in HepG2 cell cultures. The detailed mechanism needs further investigation. 


\section{Acknowledgements:}

Gerd Nilsson and Siv Svensson provided excellent technical assistance. This work was supported by grants from the Physiographic Society of Lund, the Lars Hiertas Foundation, the Foundation of Lunds University Hospital, the Fredrik and Ingrid Thurings Foundation, the OE \& Edla Johansson's Scientific Foundation, the Crafoords Foundation, Region Skåne and the Swedish Research Council (project no. 6834 and 4966). 


\section{References}

[1] N. Xu, B. Dahlback, A novel human apolipoprotein (apoM), J Biol Chem 274 (1999) 31286-90.

[2] G. Luo, X. Zhang, P. Nilsson-Ehle, N. Xu, Apolipoprotein M, Lipids Health Dis 3 (2004) 21.

[3] X.Y. Zhang, X. Dong, L. Zheng, G.H. Luo, Y.H. Liu, U. Ekstrom, P. NilssonEhle, Q. Ye, N. Xu, Specific tissue expression and cellular localization of human apolipoprotein $\mathrm{M}$ as determined by in situ hybridization, Acta Histochem 105 (2003) 67-72.

[4] X.Y. Zhang, G.Q. Jiao, M. Hurtig, X. Dong, L. Zheng, G.H. Luo, P. Nilsson-Ehle, Q. Ye, N. Xu, Expression pattern of apolipoprotein M during mouse and human embryogenesis, Acta Histochem 106 (2004) 123-8.

[5] C. Wolfrum, M.N. Poy, M. Stoffel, Apolipoprotein M is required for prebetaHDL formation and cholesterol efflux to HDL and protects against atherosclerosis, Nat Med 11 (2005) 418-22.

[6] N. Xu, P. Nilsson-Ehle, M. Hurtig, B. Ahren, Both leptin and leptin-receptor are essential for apolipoprotein M expression in vivo, Biochem Biophys Res Commun 321 (2004) 916-21.

[7] N. Xu, P. Nilsson-Ehle, B. Ahren, Correlation of apolipoprotein M with leptin and cholesterol in normal and obese subjects, J Nutr Biochem 15 (2004) 579-82.

[8] N. Xu, U. Ekstrom, P. Nilsson-Ehle, Acth decreases the expression and secretion of apolipoprotein b in hepg2 cell cultures, J Biol Chem 276 (2001) 38680-4.

[9] N. Xu, X.Y. Zhang, X. Dong, U. Ekstrom, Q. Ye, P. Nilsson-Ehle, Effects of Platelet-Activating Factor, Tumor Necrosis Factor, and Interleukin-1alpha on the Expression of Apolipoprotein M in HepG2 Cells, Biochem Biophys Res Commun 292 (2002) 944-50.

[10] N. Xu, M. Hurtig, X.Y. Zhang, Q. Ye, P. Nilsson-Ehle, Transforming growth factor-beta down-regulates apolipoprotein M in HepG2 cells, Biochim Biophys Acta 1683 (2004) 33-7.

[11] N. Xu, M. Hurtig, U. Ekstrom, P. Nilsson-Ehle, Adrenocorticotrophic hormone retarded metabolism of low-density lipoprotein in rats, Scand J Clin Lab Invest 64 (2004) 217-22.

[12] P. Chomczynski, N. Sacchi, Single-step method of RNA isolation by acid guanidinium thiocyanate-phenol-chloroform extraction, Anal Biochem 162 (1987) 156-9.

[13] N. Dashti, G. Wolfbauer, Secretion of lipids, apolipoproteins, and lipoproteins by human hepatoma cell line, HepG2: effects of oleic acid and insulin, J Lipid Res 28 (1987) 423-36.

[14] C.S. Au, A. Wagner, T. Chong, W. Qiu, J.D. Sparks, K. Adeli, Insulin regulates hepatic apolipoprotein B production independent of the mass or activity of Akt1/PKBalpha, Metabolism 53 (2004) 228-35.

[15] M.M. van Greevenbroek, V.M. Vermeulen, T.W. de Bruin, Familial combined hyperlipidemia plasma stimulates protein secretion by HepG2 cells: identification of fibronectin in the differential secretion proteome, J Lipid Res 43 (2002) 184654. 
[16] N.M. Borradaile, L.E. de Dreu, M.W. Huff, Inhibition of net HepG2 cell apolipoprotein $\mathrm{B}$ secretion by the citrus flavonoid naringenin involves activation of phosphatidylinositol 3-kinase, independent of insulin receptor substrate-1 phosphorylation, Diabetes 52 (2003) 2554-61.

[17] N. Dashti, D.L. Williams, P. Alaupovic, Effects of oleate and insulin on the production rates and cellular mRNA concentrations of apolipoproteins in HepG2 cells, Journal of Lipid Research 30 (1989) 1365-73.

[18] J.D. Sparks, T.L. Phung, M. Bolognino, C.E. Sparks, Insulin-mediated inhibition of apolipoprotein B secretion requires an intracellular trafficking event and phosphatidylinositol 3-kinase activation: studies with brefeldin A and wortmannin in primary cultures of rat hepatocytes, Biochem J 313 ( Pt 2) (1996) 567-74.

[19] S. Han, J.D. Ritzenthaler, B. Wingerd, J. Roman, Activation of peroxisome proliferator-activated receptor beta/delta (PPARbeta/delta) increases the expression of prostaglandin E2 receptor subtype EP4. The roles of phosphatidylinositol 3-kinase and CCAAT/enhancer-binding protein beta, J Biol Chem 280 (2005) 33240-9.

[20] P.D. Zenobi, S.E. Jaeggi-Groisman, W.F. Riesen, M.E. Roder, E.R. Froesch, Insulin-like growth factor-I improves glucose and lipid metabolism in type 2 diabetes mellitus, J Clin Invest 90 (1992) 2234-41.

[21] P.D. Zenobi, P. Holzmann, Y. Glatz, W.F. Riesen, E.R. Froesch, Improvement of lipid profile in type 2 (non-insulin-dependent) diabetes mellitus by insulin-like growth factor I, Diabetologia 36 (1993) 465-9.

[22] E.R. Froesch, P.D. Zenobi, M. Hussain, Metabolic and therapeutic effects of insulin-like growth factor I, Horm Res 42 (1994) 66-71.

[23] P.D. Zenobi, S. Graf, H. Ursprung, E.R. Froesch, Effects of insulin-like growth factor-I on glucose tolerance, insulin levels, and insulin secretion, J Clin Invest 89 (1992) 1908-13.

[24] C.R. Pullinger, J.D. North, B.B. Teng, V.A. Rifici, A.E. Ronhild de Brito, J. Scott, The apolipoprotein B gene is constitutively expressed in HepG2 cells: regulation of secretion by oleic acid, albumin, and insulin, and measurement of the mRNA half-life, J Lipid Res 30 (1989) 1065-77.

[25] A. Theriault, R. Cheung, K. Adeli, Expression of apolipoprotein B in vitro in cellfree lysates of HepG2 cells: evidence that insulin modulates ApoB synthesis at the translational level, Clin Biochem 25 (1992) 321-3.

[26] K. Adeli, A. Theriault, Insulin modulation of human apolipoprotein B mRNA translation: studies in an in vitro cell-free system from HepG2 cells, Biochem Cell Biol 70 (1992) 1301-12.

[27] P. Gervois, I.P. Torra, J.C. Fruchart, B. Staels, Regulation of lipid and lipoprotein metabolism by PPAR activators, Clin Chem Lab Med 38 (2000) 3-11.

[28] V. Clavey, C. Copin, M.C. Mariotte, E. Bauge, G. Chinetti, J. Fruchart, J.C. Fruchart, J. Dallongeville, B. Staels, Cell culture conditions determine apolipoprotein CIII secretion and regulation by fibrates in human hepatoma HepG2 cells, Cell Physiol Biochem 9 (1999) 139-49.

[29] B. Staels, J. Dallongeville, J. Auwerx, K. Schoonjans, E. Leitersdorf, J.C. Fruchart, Mechanism of action of fibrates on lipid and lipoprotein metabolism, Circulation 98 (1998) 2088-93. 
[30] S.P. Tam, Effects of gemfibrozil and ketoconazole on human apolipoprotein AI, $\mathrm{B}$ and $\mathrm{E}$ levels in two hepatoma cell lines, HepG2 and Hep3B, Atherosclerosis 91 (1991) 51-61.

[31] T. Fu, D. Mukhopadhyay, N.O. Davidson, J. Borensztajn, The peroxisome proliferator-activated receptor alpha (PPARalpha) agonist ciprofibrate inhibits apolipoprotein B mRNA editing in low density lipoprotein receptor-deficient mice: effects on plasma lipoproteins and the development of atherosclerotic lesions, J Biol Chem 279 (2004) 28662-9.

[32] D. Linden, K. Lindberg, J. Oscarsson, C. Claesson, L. Asp, L. Li, M. Gustafsson, J. Boren, S.O. Olofsson, Influence of peroxisome proliferator-activated receptor alpha agonists on the intracellular turnover and secretion of apolipoprotein (Apo) B-100 and ApoB-48, J Biol Chem 277 (2002) 23044-53.

[33] S.P. Anderson, P. Howroyd, J. Liu, X. Qian, R. Bahnemann, C. Swanson, M.K. Kwak, T.W. Kensler, J.C. Corton, The transcriptional response to a peroxisome proliferator-activated receptor alpha (PPAR alpha) agonist includes increased expression of proteome maintenance genes, J Biol Chem (2004). 


\section{Legend to figures:}

Fig. 1. Real-time PT-PCR determination of apoM levels in control cells and in insulin treated cells. HepG2 cells were grown in the culture medium with different concentrations of insulin for 24 hrs. Each experimental group contains 6 replicates and real-time RT-PCR was run in triplicate. Data are mean \pm S.D. The control group represented $100 \%$. ${ }^{* * *} \mathrm{P}<0.001$ vs. control group.

Fig. 2. Real-time PT-PCR determination of apoM levels in control cells and in IGF-I treated cells. HepG2 cells were grown in the culture medium with different concentrations of IGF-I for 24 hrs. Each experimental group contains 6 replicates and real-time RT-PCR was run in triplicate. Data are mean \pm S.D. The control group represented $100 \%$. * $\mathrm{P}<0.05 ; * * * \mathrm{P}<0.001$ vs. control group.

Fig. 3. Real-time PT-PCR determination of apoM levels in control cells and in IGF-IPP treated cells. HepG2 cells were grown in the culture medium with different concentrations of IGF-IPP for 24 hrs. Each experimental group contains 6 replicates and real-time RT-PCR was run in triplicate. Data are mean \pm S.D. The control group represented $100 \%$. ** $\mathrm{P}<0.01$ vs. control group.

Fig. 4. Time course of insulin on apoM expression. HepG2 cells were grown in the culture medium with (10 $\mu \mathrm{g} / \mathrm{ml}$ insulin) or without insulin (control). ApoM mRNA levels were determined by real-time RT-PCR. Each experimental group contains three 
replicates and real-time RT-PCR was run triplicates. Control represented 100\%. Data are mean \pm S.D.

Fig. 5. Effect of AG1024 on insulin-induced inhibition of apoM expression. Cells were incubated with $10 \mu \mathrm{g} / \mathrm{ml}$ insulin or without insulin (control) for $3 \mathrm{hrs}$ and then add $10 \mu \mathrm{M}$ AG1024 for another 3 hrs. Each experimental group contains 3-6 replicates and real-time RT-PCR was run in triplicate. Data are mean \pm S.D. The control group represented $100 \%$. $* \mathrm{P}<0.05$ vs. control group.

Fig. 6. Effect of LY294002 on insulin-induced inhibition of apoM expression. Cells were incubated with $10 \mu \mathrm{g} / \mathrm{ml}$ insulin in present different concentrations of LY294002 or absent LY294002 (control) for 3 hrs. Each experimental group contains 6 replicates and real-time RT-PCR was run in triplicate. Data are mean \pm S.D. The control group (with insulin but without LY294002) represented 100\%. * $\mathrm{P}<0.05$; *** $\mathrm{P}<0.001$ vs. control group.

Fig. 7. Effects of PPAR- $\alpha$ agonist (GW7647) or PPAR- $\gamma$ agonist (GW1929) on apoB and apoM expression. HepG2 cells were grown in the culture medium with different amounts of GW7647 or GW1929 for 24 hrs. ApoM mRNA levels were determined by real-time RT-PCR. Each experimental group contains four to six replicates and real-time RT-PCR was run triplicates. Control represented $100 \%$. Data are mean \pm S.D. ${ }^{* *} \mathrm{p}<0.01 ;{ }^{* * *} \mathrm{p}<$ 0.001, vs. control. 
Fig. 8. Effects of PPAR- $\beta / \delta$ agonist (GW501516) on apoB and apoM expression. HepG2 cells were grown in the culture medium with different amounts of GW501516 for 24 hrs. ApoB and apoM mRNA levels were determined by real-time RT-PCR. Each experimental group contains six replicates and real-time RT-PCR was run triplicates. Control represented 100\%. Data are mean \pm S.D. ${ }^{*} \mathrm{p}<0.05 ;{ }^{* *} \mathrm{p}<0.01$, vs. control. 
Fig. 1

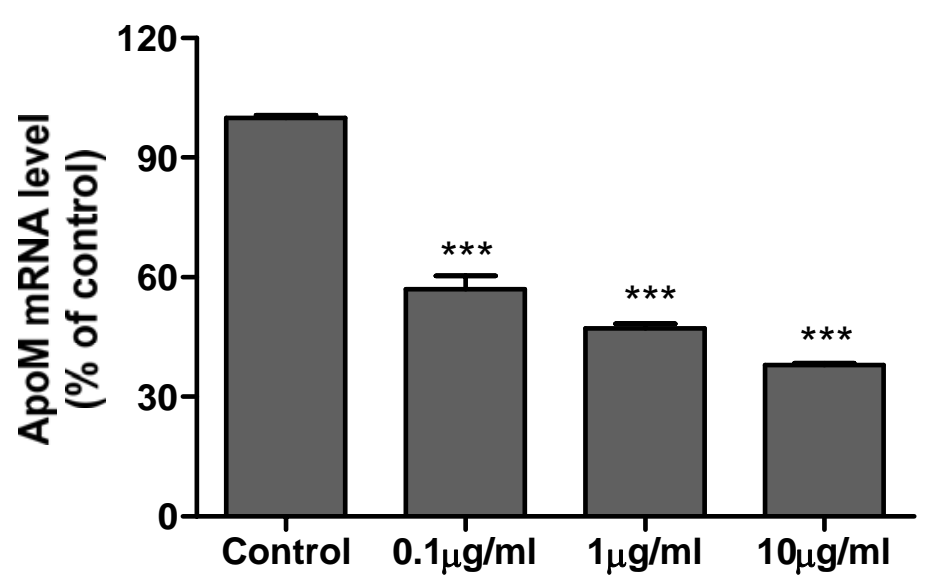


Fig. 2

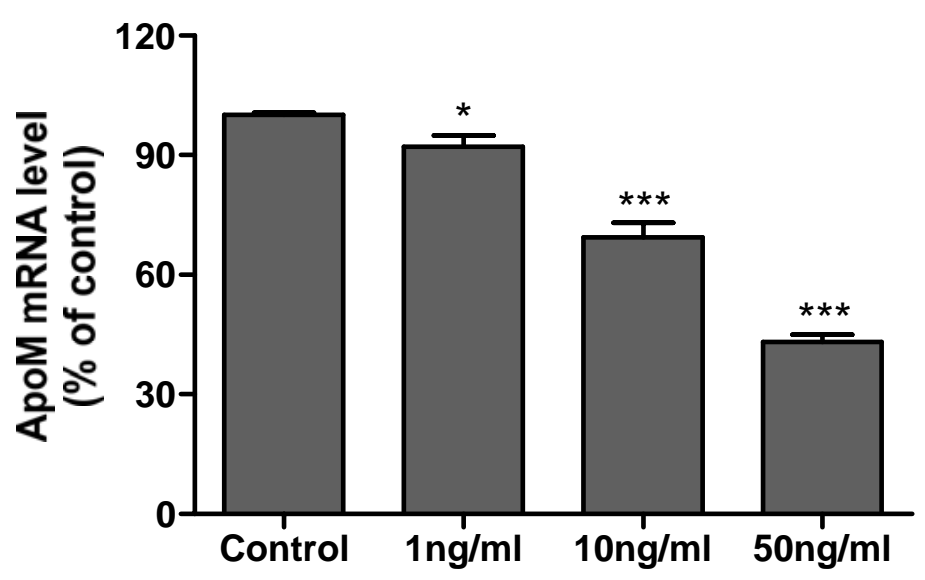


Fig. 3

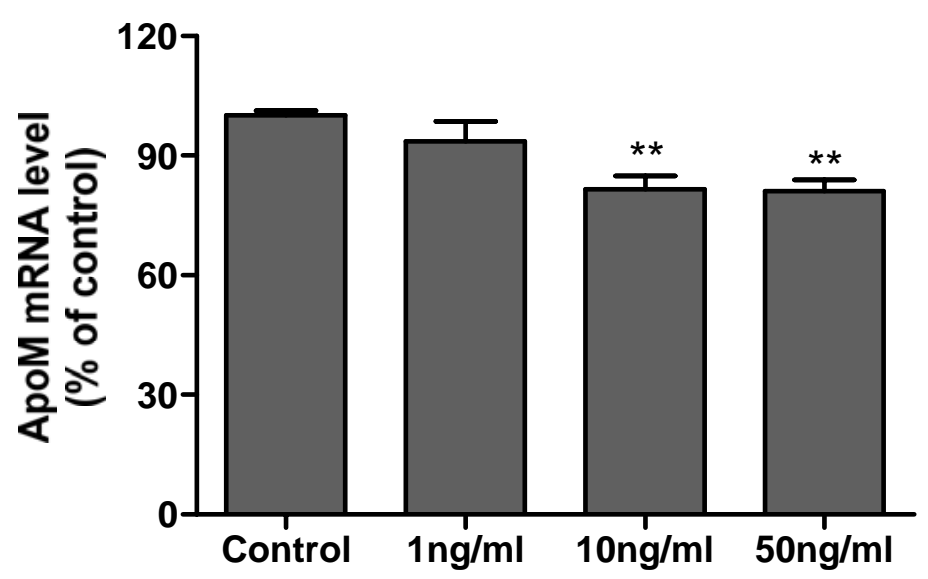


Fig. 4

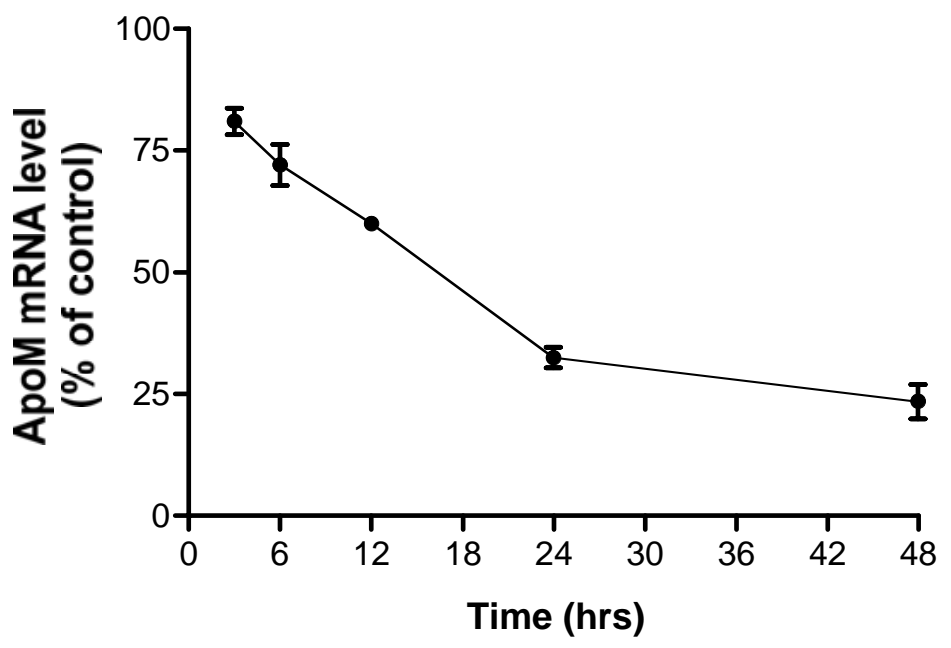


Fig. 5

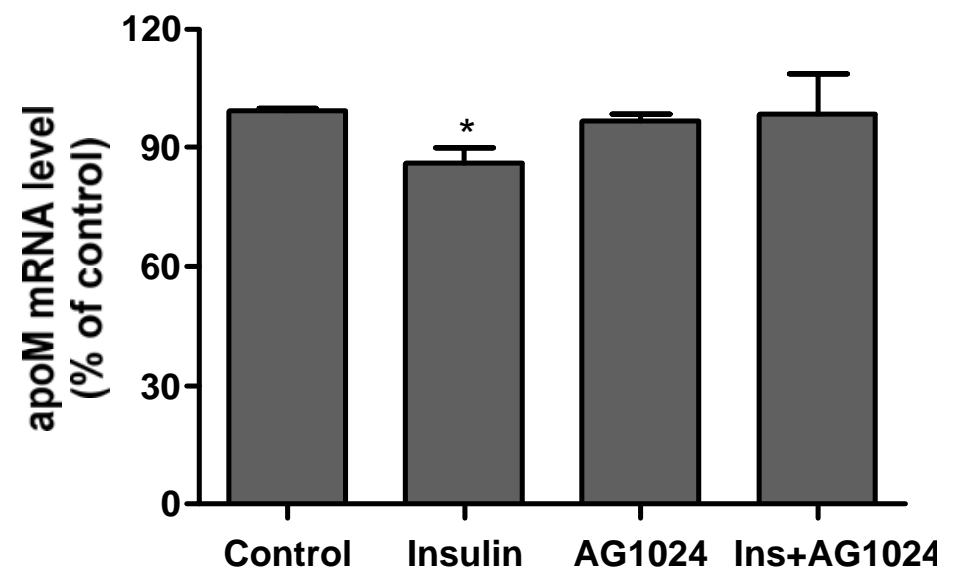


Fig. 6

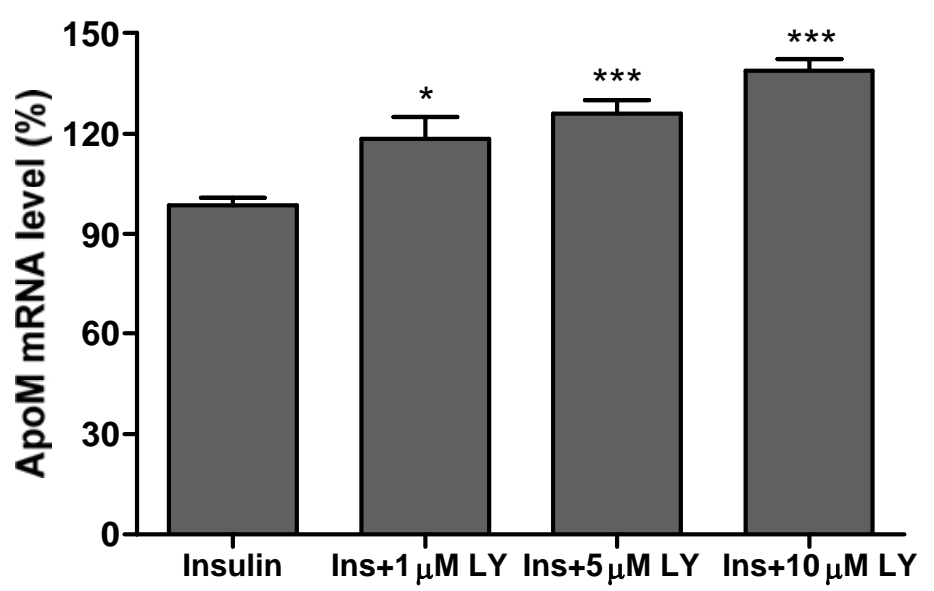


Fig. 7

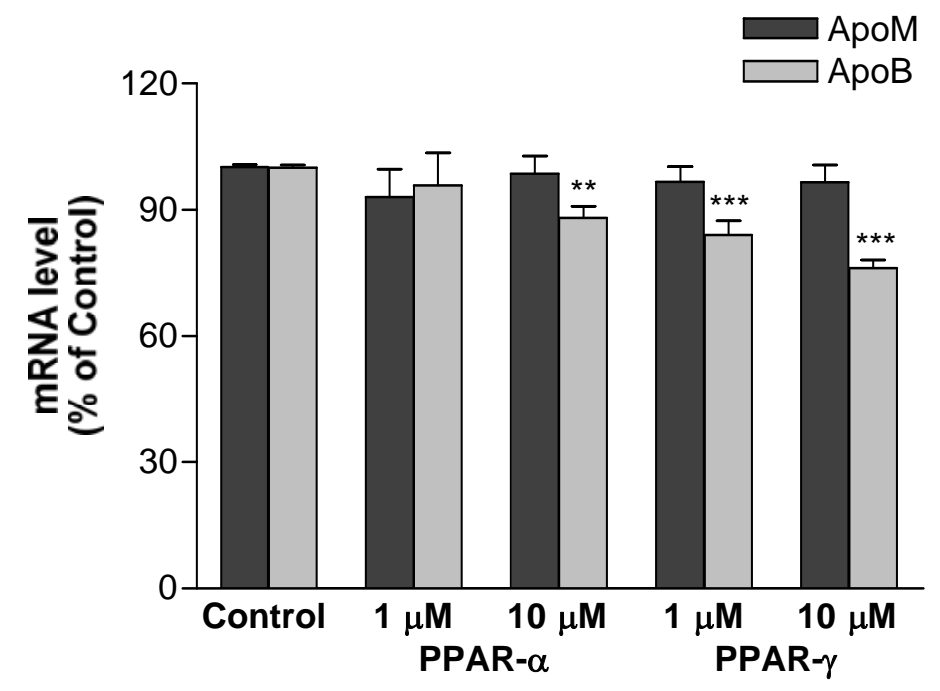


Fig. 8

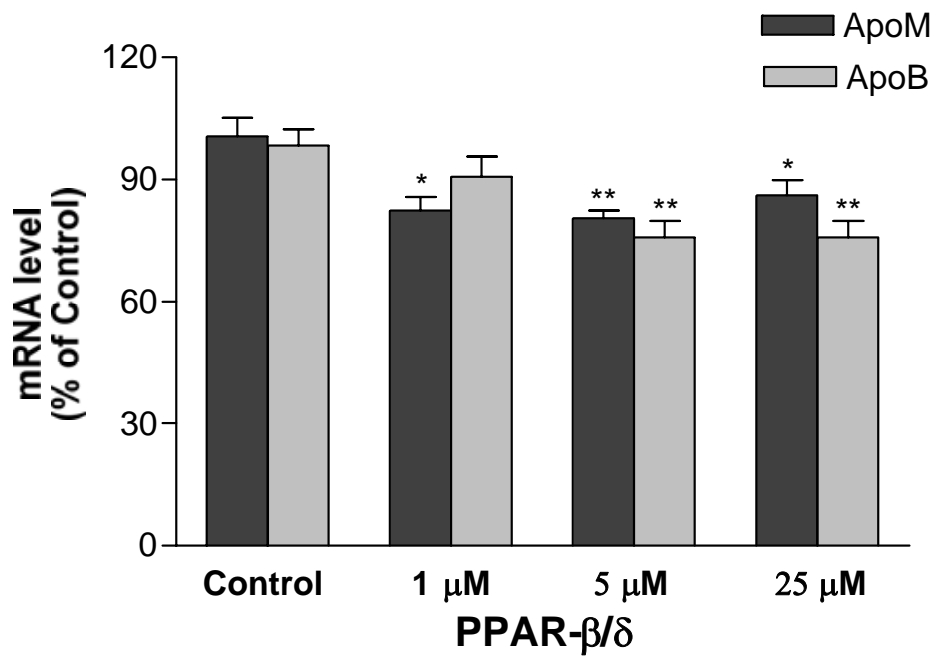

